\title{
Downregulation of leucine-rich- $\alpha$-2-glycoprotein 1 expression is associated with the tumorigenesis of head and neck squamous cell carcinoma
}

\author{
YONGPING WANG ${ }^{1 *}$, CHEN CHEN $^{2 *}$, QINGQUAN HUA ${ }^{1}$, LEI WANG ${ }^{1}$, FEN LI $^{2}$, MAN LI $^{1}$, \\ ZHIDAN MEI ${ }^{1}$, TAO ZHOU ${ }^{1}$, BOKUI XIAO ${ }^{2}$ and ZEZHANG TAO ${ }^{1,2}$ \\ ${ }^{1}$ Department of Otolaryngology-Head and Neck Surgery, and ${ }^{2}$ Research Institute of Otolaryngology-Head and Neck Surgery, \\ Renmin Hospital of Wuhan University, Wuhan, Hubei 430060, P.R. China
}

Received June 1, 2016; Accepted November 15, 2016

DOI: $10.3892 /$ or.2017.5377

\begin{abstract}
Leucine-rich- $\alpha$-2-glycoprotein 1 (LRG1) is considered as a potential biomarker as it is aberrantly expressed in various malignancies. However, there is limited information regarding its role in head and neck squamous cell carcinoma (HNSCC). In the present study, we aimed to explore the expression pattern of LRG1 in HNSCC and its clinicopathological significance. We first analyzed LRG1 gene expression in HNSCC by investigating data obtained from the Gene Expression Omnibus (GEO) database. The results showed that LRG1 was downregulated in HNSCC tissues and its expression level was negatively related to tumor $\mathrm{T}$ and $\mathrm{N}$ stages and degree of malignancy. Then, we further tested a tissue microassay and clinical samples, respectively, by immunohistochemical staining and western blotting. Consistently, the results revealed that LRG1 expression was decreased in tumor tissues regardless of the grade of the tumor. Moreover, the protein level of LRG1 showed slight differences among four $\mathrm{T}$ stages or three $\mathrm{N}$ stages. In addition, there were no significant associations between LRG1 protein expression and other clinicopathological parameters such as gender, age, tumor location and clinical staging. These findings imply that downregulation of the expression of LRG1 is correlated with tumorigenesis but not with the development of HNSCC, indicating the potential clinical value of LRG1 in the early diagnosis of HNSCC.
\end{abstract}

\section{Introduction}

Head and neck squamous cell carcinoma (HNSCC) has the sixth highest incidence rate worldwide among all common

Correspondence to: Professor Zezhang Tao, Department of Otolaryngology-Head and Neck Surgery, Renmin Hospital of Wuhan University, 238 Jiefang Road, Wuhan, Hubei 430060, P.R. China

E-mail: taozezhang@hotmail.com

*Contributed equally

Key words: head and neck squamous cell carcinoma, GEO dataset, LRG1, tumorigenesis, biomarker cancers (1). Despite the advances in oncology and multidisciplinary treatment, the recurrence rate and mortality of HNSCC have not obviously declined. One important reason is that more than $50 \%$ of HNSCC patients present with advanced stage at the time of diagnosis $(2,3)$. Therefore there is a critical need to find effective pathways by which to predict tumor genesis and development. Recently, various proteins have been found to be closely correlated with the occurrence and progression of HNSCC $(4,5)$. However, new molecules which possess diagnostic value for clinical application are still needed to be discovered.

Leucine-rich- $\alpha$-2-glycoprotein 1 (LRG1), a member of the leucine-rich repeat (LRR) family proteins, was initially isolated from human serum by Haupt and Baudner in 1977 (6). The LRR family proteins are classified as secreted proteins, characterized by the presence of leucine-rich repeats in amino acid residues, and have 20-29-residue sequence motifs containing a conserved 11-residue segment (7). These proteins were reported to be involved in several important biological processes, such as hormone-receptor interactions, enzyme inhibition, cell adhesion and cellular trafficking (8). LRG1 is considered as a membrane-associated protein, consisting of 312 amino acid residues, 66 of which are leucines. To date, the biological function of LRG1 has not been fully elicidated. It has been reported to be an inflammatory protein involved in inflammatory responses such as active ulcerative colitis (9), acute appendicitis (10) and active rheumatoid arthritis (11). Elevated LRG1 expression may be induced by proinflammatory cytokines such as interleukin-6 (IL-6) (9). Evidence has also shown that LRG1 participates in the regulatory mechanism of aberrant angiogenesis by modulating endothelial TGF- $\beta$ signaling (12). Aberrant neovascularization contributes to tumor growth, and LRG1 was found to be a biomarker and is upregulated in various types of carcinomas, such as hepatocellular carcinoma (13), ovarian cancer (14), endometrial carcinoma (15), gastric cancer (16), leukemia (17), colorectal (18), pancreatic (19) and bladder cancer (20). In addition, LRG1 is involved in tumor development, progression and metastasis and is regarded as an indicator of tumor prognosis. However, the role of LRG1 in the tumorigenesis and progression of HNSCC is not yet clear, and warrants elucidation. 
Table I. Detailed information of the GEO datasets in the present study.

\begin{tabular}{|c|c|c|c|}
\hline Series accession & Organism & Type & Platform \\
\hline GSE51985 & Homo sapiens & Expression profiling by array & $\begin{array}{l}\text { GPL10588 Illumina HumanHT-12 v4.0 } \\
\text { expression BeadChip }\end{array}$ \\
\hline GSE33205 & Homo sapiens & Expression profiling by array & GPL5175 Affymetrix Human Exon 1.0 ST Array \\
\hline GSE59102 & Homo sapiens & Expression profiling by array & $\begin{array}{l}\text { GPL6480 Agilent-014850 Whole Human } \\
\text { Genome Microarray 4X44K G4112F }\end{array}$ \\
\hline GSE58911 & Homo sapiens & Expression profiling by array & GPL6244 Affymetrix Human Gene 1.0 ST Array \\
\hline GSE13399 & Homo sapiens & Expression profiling by array & $\begin{array}{l}\text { GPL7540 Agilent-scanner-UNC-custom- } \\
\text { 4X44K-without-Virus }\end{array}$ \\
\hline GSE39366 & Homo sapiens & Expression profiling by array & GPL9053 Agilent-UNC-custom-4X44K \\
\hline
\end{tabular}

GEO, Gene Expression Omnibus.

In the present study, we first investigated the LRG1 gene expression pattern in HNSCC by analysis of the data obtained from Gene Expression Omnibus (GEO) datasets, and its relationships with degree of malignancy, tumor stage and regional lymph node metastasis were explored, respectively. Secondly, tissue microassay and clinical samples were investigated for further confirmation of the findings.

\section{Materials and methods}

Gene expression profiles. LRG1 expression data were retrieved from Gene Expression Omnibus (GEO) (http://www.ncbi.nlm. nih.gov/geo/). Six datasets were obtained for the analysis including GSE51985 [10 primary laryngeal squamous cell carcinoma (LSCC) and corresponding adjacent non-neoplastic tissues were analyzed], GSE33205 (analysis of 44 cases of HNSCC tumors and 25 cases of uvupopalatopharyngoplasty patients as control), GSE59102 (29 LSCC samples and 13 marginal samples were collected for microarray analysis), GSE58911 (15 paired normal and HNSCC samples from individual patients were analyzed), GSE13399 (16 paired HNSCC tumor samples and normal tonsil samples were collected) and GSE39366 (a total of 163 samples were considered and a total of 138 tumor arrays remained after removing low-quality and duplicate arrays, and arrays from non-HNSCC samples). More details of the series data are listed in Table I. The expression values of the LRG1 gene were transformed to the relative expression.

Tissue microassay and clinical samples. The HNSCC tissue microassay (TMA) was procured from US Biomax Co. (Rockville, MD, USA) (production no. HN803b). Eleven cases of normal tissue, 31 cases of tongue carcinoma, 31 larynx carcinoma cases and 7 nose carcinoma cases were contained in the TMA, including 62 men and 18 women. The mean age was 53.4 years (range 18-90 years). More details are listed in Table II.

Twenty pairs of HNSCC tissues including 18 cases of larynx carcinoma and 2 cases of hypopharyngeal carcinoma and their corresponding para-carcinoma normal tissues were collected between 2012 and 2013 from the Renmin Hospital of Wuhan University. Baseline clinical features are described in detail in Table III. All tissues were frozen at $-125^{\circ} \mathrm{C}$ until proteins were extracted for western blotting.

The present study was approved by the appropriate Ethics Committees related to the institutions in which it was performed. The authors assert that all procedures contributing to the present study comply with the ethical standards of the relevant national and institutional guidelines.

Immunohistochemical staining. To analyze LRG1 expression by immunohistochemistry (IHC), the TMA was deparaffined in xylene for 15 min three times at room temperature, hydrated in a series of 100, 95, 90, 80, 70 and $60 \%$ ethanol solutions, and washed in phosphate-buffered saline (PBS). The antigen was recovered in boiling citrate buffer $(10 \mathrm{mmol} / 1$, $\mathrm{pH}$ 6.0) for $15 \mathrm{~min}$ and then the sections were cooled down to room temperature. To quench endogenous peroxidase activity, the sections were incubated with $0.3 \%$ hydrogen peroxide phosphate-citrate buffer for $10 \mathrm{~min}$ and then rinsed extensively in PBS. The sections were incubated with primary rabbit anti-LRG1 monoclonal antibody (dilution 1:100; Sigma-Aldrich, St. Louis, MO, USA) overnight at $4^{\circ} \mathrm{C}$. After washing with PBS, the slides were incubated with poly-HRP goat anti-rabbit (Maixin-Bio, Fuzhou, China) for $30 \mathrm{~min}$. Diaminobenzidine was used to dye the slides for $5 \mathrm{~min}$ and hematoxylin for counterstaining the nuclei. The sections were then dehydrated in ethanol and cleared in xylene. Coverslips were placed on the slides. Images of sections were captured using an Olympus BX40 microscope and CC-12 Soft-Imaging system (Olympus, Tokyo, Japan).

Evaluation of immunohistochemical staining. LRG1-positive cells displayed brownish yellow granules on the cytoplasm and/or the membrane. Evaluation of LRG1 staining included the intensity of staining (scored as: 0 , no staining; 1 , weak staining; 2 , moderate staining; and 3 , strong staining) and the percentage of positive tumor cells (scored as: $0,<5 \%$; $1,5-25 \% ; 2,26-50 \% ; 3,51-75 \%$; and 4, 76-100\%). To facilitate the statistical analysis, LRG1 staining intensity and frequency were transformed into a Composite Expression Score (CES) utilizing the formula: CES = Intensity x Frequency. The range of CES was from 0 to 12 . The CES was scored as: 0, negative; 1-4, weak positive; $5-8$, positive; $9-12$, strong positive. 
Table II. Immunohistochemistry staining of LRG1 and its correlation with clinicopathological features of the HNSCC cases.

\begin{tabular}{|c|c|c|c|}
\hline $\begin{array}{l}\text { Clinicopathological } \\
\text { parameters }\end{array}$ & $\begin{array}{l}\text { No. of } \\
\text { pts. }(\%)\end{array}$ & $\begin{array}{l}\text { LRG1 expression } \\
\text { score }(\mathrm{CES}) \text { : } \\
\text { mean } \pm \mathrm{SD}\end{array}$ & P-value \\
\hline Gender & & & 0.2027 \\
\hline Male & $57(82.6)$ & $4.54 \pm 2.89$ & \\
\hline Female & $12(17.4)$ & $3.12 \pm 2.80$ & \\
\hline Age (years) & & & 0.3387 \\
\hline$\leq 60$ & $42(60.9)$ & $4.63 \pm 2.79$ & \\
\hline$>60$ & $27(39.1)$ & $3.86 \pm 3.07$ & \\
\hline Location & & & 0.6628 \\
\hline Larynx & $31(44.9)$ & $4.75 \pm 2.51$ & \\
\hline Nose & $7(10.1)$ & $4.00 \pm 3.10$ & \\
\hline Tongue & $31(44.9)$ & $4.04 \pm 3.23$ & \\
\hline Histological grade ${ }^{a}$ & & & 0.0613 \\
\hline Well & $10(15.6)$ & $3.00 \pm 2.14$ & \\
\hline Moderate & $34(53.1)$ & $4.45 \pm 2.56$ & \\
\hline Poor & $20(31.5)$ & $5.60 \pm 2.40$ & \\
\hline T stage & & & 0.2470 \\
\hline $\mathrm{T} 1$ & $5(8.2)$ & $3.75 \pm 1.71$ & \\
\hline $\mathrm{T} 2$ & $33(54.1)$ & $4.00 \pm 2.54$ & \\
\hline $\mathrm{T} 3$ & $14(23.0)$ & $5.78 \pm 2.11$ & \\
\hline $\mathrm{T} 4$ & $9(14.8)$ & $3.88 \pm 2.64$ & \\
\hline $\mathrm{N}$ stage & & & 0.1935 \\
\hline No & $43(70.5)$ & $3.89 \pm 2.82$ & \\
\hline N1 & $15(24.6)$ & $4.17 \pm 2.33$ & \\
\hline $\mathrm{N} 2$ & $3(4.9)$ & $7.50 \pm 2.12$ & \\
\hline Clinical stage & & & 0.1710 \\
\hline Early I-II & $28(45.9)$ & $3.56 \pm 2.83$ & \\
\hline Advanced III-IV & $33(54.1)$ & $4.62 \pm 2.59$ & \\
\hline Metastasis $^{\mathrm{b}}$ & $8(10)$ & $6.80 \pm 3.63$ & NA \\
\hline Normal & $11(13.8)$ & $7.10 \pm 3.10$ & NA \\
\hline
\end{tabular}

${ }^{a}$ Five cases had no data for histological grade. ' Includes two cases of acinic cell carcinoma and two cases of mucoepidermoid carcinoma. HNSCC, head and neck squamous cell carcinoma; pts., patients; LRG1, leucine-rich- $\alpha$-2-glycoprotein 1; CES, Composite Expression Score; NA, not available.

These scores were independently determined by two senior pathologists.

Protein extraction. Frozen HNSCC and corresponding para-carcinoma tissues were cut into $50-100 \mathrm{mg}$ fragments and ground using a homogenizer containing liquid nitrogen, then lysed in ice-cold RIPA buffer [50 mM Tris- $\mathrm{HCl} \mathrm{pH} 7.4$, $150 \mathrm{mM} \mathrm{NaCl}, 1 \%$ Triton X-100, 0.25\% deoxycholate (DOC), $1.5 \mathrm{mM} \mathrm{MgCl}_{2}, 1 \mathrm{mM}$ EGTA, $1 \mathrm{mM}$ phenylmethyl sulfonylfluoride (PMSF), $10 \mathrm{mM} \mathrm{NaF}, 10 \mathrm{mM}$ pervanadate, $10 \mu \mathrm{g}$ / $\mathrm{ml}$ leupeptin and $10 \mu \mathrm{g} / \mathrm{ml}$ aprotinin], and incubated on ice with intermittent vortexing for $30 \mathrm{~min}$. Insoluble cellular
Table III. Clinicopathological features of the HNSCC patients employed in western blotting.

\begin{tabular}{|c|c|}
\hline Clinicopathological parameters & No. of patients \\
\hline Gender (male:female) & $20(19: 1)$ \\
\hline Age, years, mean \pm SD & $59.8 \pm 7.8$ \\
\hline \multicolumn{2}{|l|}{ Tobacco smoking } \\
\hline Yes & 11 \\
\hline No & 9 \\
\hline \multicolumn{2}{|l|}{ Alcohol consumption } \\
\hline Yes & 6 \\
\hline No & 14 \\
\hline \multicolumn{2}{|l|}{ Location } \\
\hline Larynx & 17 \\
\hline Hypopharynx & 3 \\
\hline \multicolumn{2}{|l|}{ Occurrence } \\
\hline Primary & 16 \\
\hline Recurrence & 4 \\
\hline \multicolumn{2}{|l|}{ Histological grade } \\
\hline Well/moderate & 16 \\
\hline Poor & 4 \\
\hline \multicolumn{2}{|l|}{ T stage } \\
\hline $\mathrm{T} 1$ & 3 \\
\hline $\mathrm{T} 2$ & 7 \\
\hline T3 & 7 \\
\hline $\mathrm{T} 4 \mathrm{a}$ & 3 \\
\hline \multicolumn{2}{|l|}{ Clinical N stage } \\
\hline cNO & 7 \\
\hline $\mathrm{cN} 1$ & 2 \\
\hline $\mathrm{cN} 2$ & 11 \\
\hline \multicolumn{2}{|l|}{ Pathological N stage } \\
\hline pNO & 12 \\
\hline $\mathrm{pN} 1$ & 2 \\
\hline $\mathrm{pN} 2$ & 6 \\
\hline Distant metastasis & 0 \\
\hline \multicolumn{2}{|l|}{ Clinical stage } \\
\hline Early I-II & 6 \\
\hline Advanced III-IV & 14 \\
\hline \multicolumn{2}{|l|}{ Neck dissection } \\
\hline Yes & 13 \\
\hline No & 7 \\
\hline Postoperative radiotherapy & 20 \\
\hline
\end{tabular}

components were removed by centrifugation for $30 \mathrm{~min}$ at $25,000 \mathrm{x} \mathrm{g} / \mathrm{min}$. The protein concentration of the supernatant lysate was determined by the BCA method. Lysate aliquots were mixed with $4 \mathrm{X}$ sample buffer containing 2-mercaptoethanol, and heated at $70^{\circ} \mathrm{C}$ for $10 \mathrm{~min}$ for western blotting.

Western blotting. For western blot analysis, protein samples were loaded on prefabricated 10\% NuPAGE Bris-Tris gel (Life Technologies, Carlsbad, CA, USA). Following separation, 
A

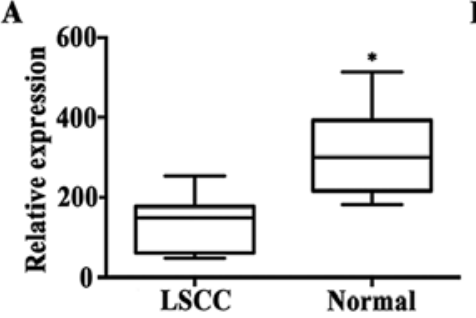

E

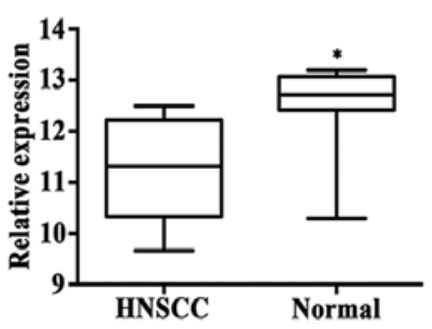

B

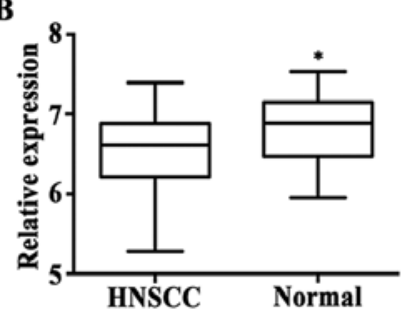

F

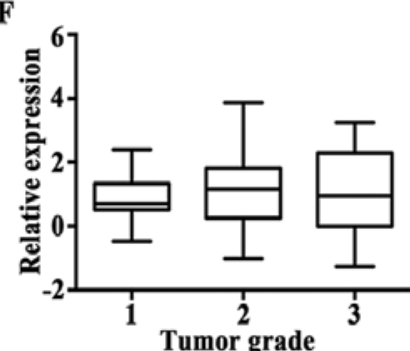

C

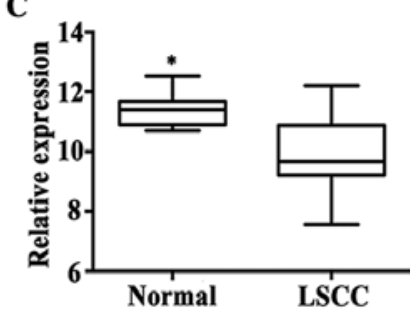

G

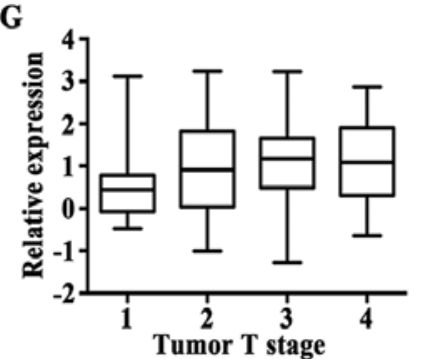

D

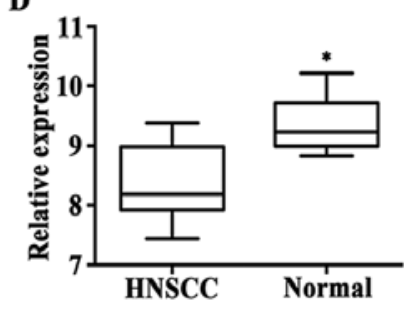

H

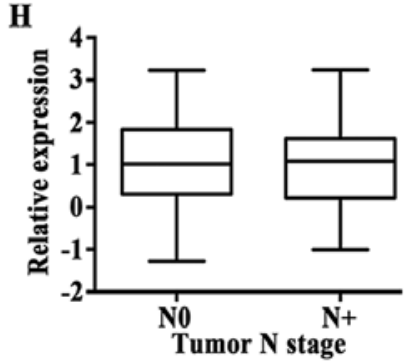

Figure 1. LRG1 gene expression analysis in HNSCC from the GEO datasets. Six datasets were retrieved from the GEO repository and further subjected to data analysis, including GSE51985, GSE33205, GSE59102, GSE58911, GSE13399 and GSE39366. (A-E) Expression of the LRG1 gene was significantly higher in non-tumor normal tissues compared with LSCC or HNSCC; *P<0.01 compared with LSCC or HNSCC. (A) GSE51985. (B) GSE33205. (C) GSE59102. (D) GSE58911. (E) GSE13399. (F) Dataset GSE39366 was analyzed to confirm that there was no significant difference in LRG1 expression between different tumor grades of HNSCC; P>0.05. (G) LRG1 gene expression in different T stages of HNSCC from series GSE39366. Analysis showed that the expression rate of LRG1 was not statistically different between each two T stage groups; $\mathrm{P}>0.05$. (H) LRG1 gene expression in different $\mathrm{N}$ stages of $\mathrm{HNSCC}$ from series GSE39366. There was no statistical significance between N0 and N+ groups; $\mathrm{P}>0.05$.

proteins were transferred to a nitrocellulose membrane in $90 \mathrm{~min}$ for LRG1 and GAPDH. After blocking with 5\% (w/v) BSA in Tris-buffer, membrane strips were incubated with a different dilution of different antibodies according to their descriptions. Anti-LRG1 and anti-GAPDH primary antibodies (Sigma-Aldrich) were respectively diluted 1:200 and 1:1,000 in $1 \mathrm{X}$ TBS, $0.1 \%$ Tween-20 with 5\% BSA. After three washes with TBS with $0.1 \%$ Tween-20, the blots were incubated 1:2,000 with horseradish peroxidase-conjugated secondary antibodies and detection was performed using enhanced chemiluminescence.

Statistical analysis. Data and figures were mainly processed using GraphPad Prism 6.0 software. Values are expressed as the mean $\pm \mathrm{SD}$ except for the intensity values in western blotting presented as the mean. Comparisons of LRG1 expression between groups were performed with independent samples t-test, or using one-way of ANOVA and Bonferroni's multiple comparison tests. A probability value $<0.05$ was considered to indicate a statistically significant result.

\section{Results}

Analysis of the GEO database. To understand the expression pattern of LRG1 in HNSCC, we first utilized GSE51985 which included 10 paired LSCC samples and corresponding adjacent non-neoplastic samples. As shown in Fig. 1A, LRG1 expression was lower in the cancer tissues compared to that in the adjacent normal tissues $(\mathrm{P}=0.0006)$. Data of other five series, containing more samples, were also analyzed, and the results showed a similar trend (all P-values $<0.01$ ). Details are shown in Fig. 1B-E. These data indicated that the LRG1 gene is downregulated in HNSCC. We further investigated whether the expression of LRG1 was related to the grade and stage of
HNSCC with series GSE39366. According to the analysis of data extraction from series GSE39366, the expression rate of LRG1 was not statistically different between tumor grade $(\mathrm{P}>0.05)$ (Fig. 1F). In addition, there was no statistical significance between each two T stage groups $(\mathrm{P}>0.05)$ (Fig. 1G) or between the two $\mathrm{N}$ stage groups $(\mathrm{P}>0.05)$ (Fig. $1 \mathrm{H})$. Taken together, the LRG1 expression status showed no correlation with HNSCC tumor differentiation and progression.

Clinicopathological characteristics of the HNSCC patients. Sixty-nine HNSCCs were included in the TMA. The location of the HNSCCs was the larynx (31 cases), the tongue (31 cases) and the nose ( 7 cases). The patient ages ranged from 32 to 90 years with a mean of 57.3 years. Fifty-seven were male, occupying a predominant position and the rest were female. Tumors categorized as T2 were the most commonly found in the TMA. Most patients were found without local lymph node metastasis (N0). The number of patients in the early stage was nearly equal to that in the advanced stage. Other details of the clinicopathological characteristics are shown in Table II.

There were 20 cases of HNSCC employed in the western blotting, including 17 cases of larynx carcinoma and 3 cases of hypopharynx carcinoma. The patient ages ranged from 41 to 72 years with a mean of 59.8 years. Nineteen patients were male, only one case was female. Half of the number had a history of smoking. T2 and T3 were frequently seen in this cohort. More than half of patients were lymph node-negative and were in the advanced stage. All patients underwent surgical therapy and most were performed with neck dissection. Most of the clinicopathological characteristics are listed in Table III.

LRG1 expression is downregulated in the HNSCC TMA. Decreased LRG1 gene expression in the HNSCC cases from the GEO dataset was found as described above. Then, we 
A

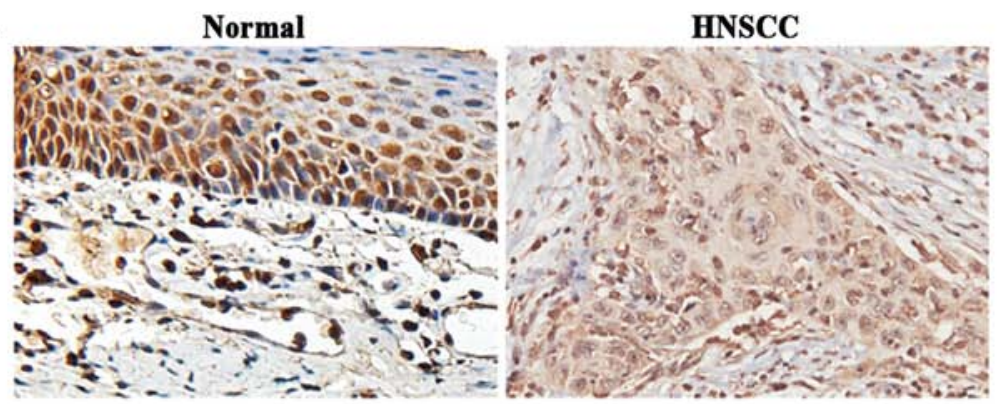

B

Figure 2. LRG1 protein expression is downregulated in the HNSCC TMA. (A) Representative images of IHC staining for LRG1 protein in tumor and normal tissues (magnification, $\mathrm{x} 400$ ). (B) A graphical representation of the mean \pm SD data represents CES of LRG1 protein staining presented in A; ${ }^{*}<0.01$ compared with the normal tissue group.

A

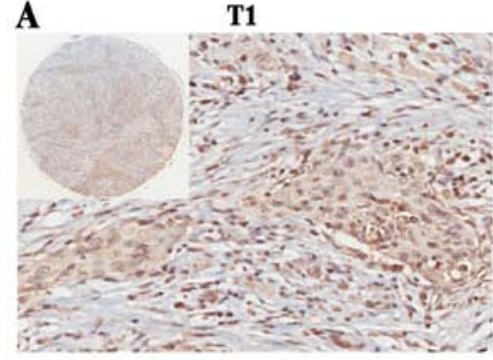

T3

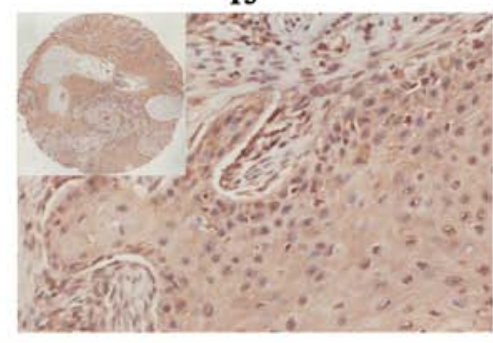

T2

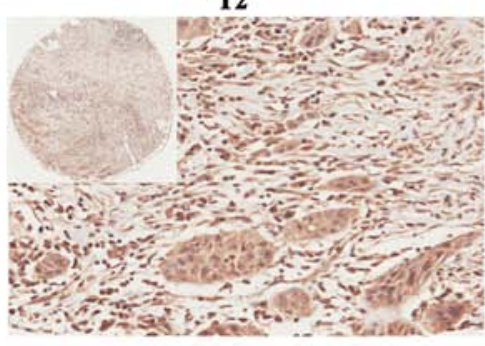

T4

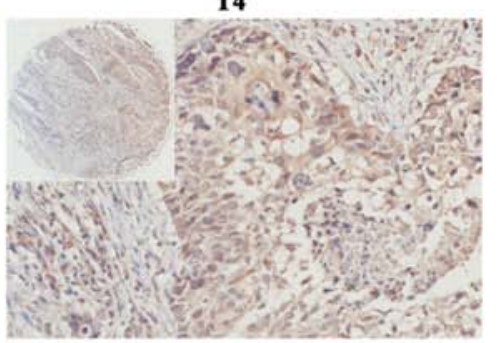

B

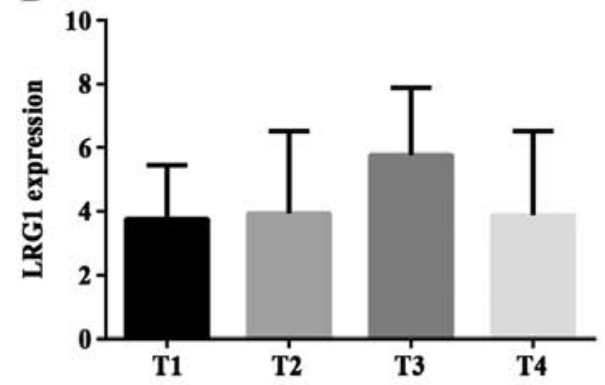

Figure 3. LRG1 protein expression in different T stages of HNSCC TMA. (A) Representative images of IHC staining in 4 T stage groups (magnification, $\mathrm{x} 400$ ). In the top left corner are original microscopic images at a magnification of $x 100$. (B) A graphical representation of the mean \pm SD data represents CES of LRG1 protein staining presented in A. There was no statistically significance between T stages; $\mathrm{P}>0.05$.

examined LRG1 protein expression in an independent cohort of 80 cases including three types of HNSCC (tongue, larynx and nose) and normal tissues on a TMA by IHC staining. CES was used for the measurement. The staining density of LRG1 in the non-cancerous tissues had a more intense coloring and broader distribution than that observed in the HNSCC tissues. Representative images of LRG1 in the tumor and normal tissues are shown in Fig. 2A. Accordingly, a significantly decrease in CES was present in the tumor tissues compared to that in the normal tissues (Fig. $2 \mathrm{~B})(\mathrm{P}<0.01)$. These results were consistent with the analysis of the GEO dataset.

LRG1 expression is not related to $T$ and $N$ stage, or differentiation of HNSCC. To further understand the correlation between LRG1 protein expression and different stages or grades, we also used IHC to analyze the specimens of the tissue chips. Representative images of LRG1 in different $\mathrm{T}$ staged tissues are shown in Fig. 3A. There was no statistical significance between $\mathrm{T}$ stage groups ( $\mathrm{P}>0.05)$ (Fig. 3B). In addition, we compared three $\mathrm{N}$ stage groups: N0, N1 and N2.
Typical images are shown in Fig. 4A, and there was no statistical difference among the groups $(\mathrm{P}>0.05)$ (Fig. 4C). These data above indicate that LRG1 protein is not involved in the development of HNSCC.

Finally, LRG1 protein expression was analyzed in different grades of tumor malignancy. Malignancy grade 1, 2 and 3 represent respectively well, moderate and poor differentiation. LRG1 staining is shown in Fig. 4B, and there was no significant difference among the groups $(\mathrm{P}>0.05)$ (Fig. 4D). This result reflected that LRG1 had no correlation with tumor grade, which was consistent with the outcome of the GEO dataset analysis.

Association of LRG1 expression and other clinicopathological characteristics in HNSCC. To further determine the clinical significance of LRG1 in HNSCC, the relationship between expression of LRG1 and other clinicopathological parameters was analyzed. In the cohort analyzed by IHC and scored by CES, there were no significant associations between LRG1 protein expression and clinicopathological parameters such as 


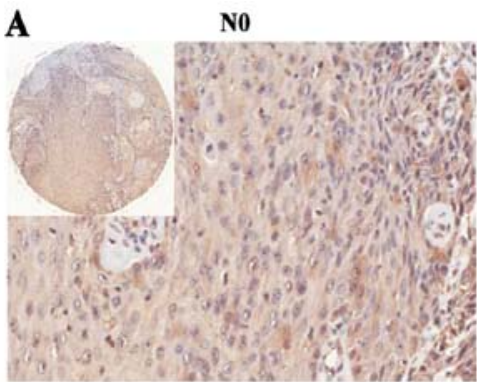

B
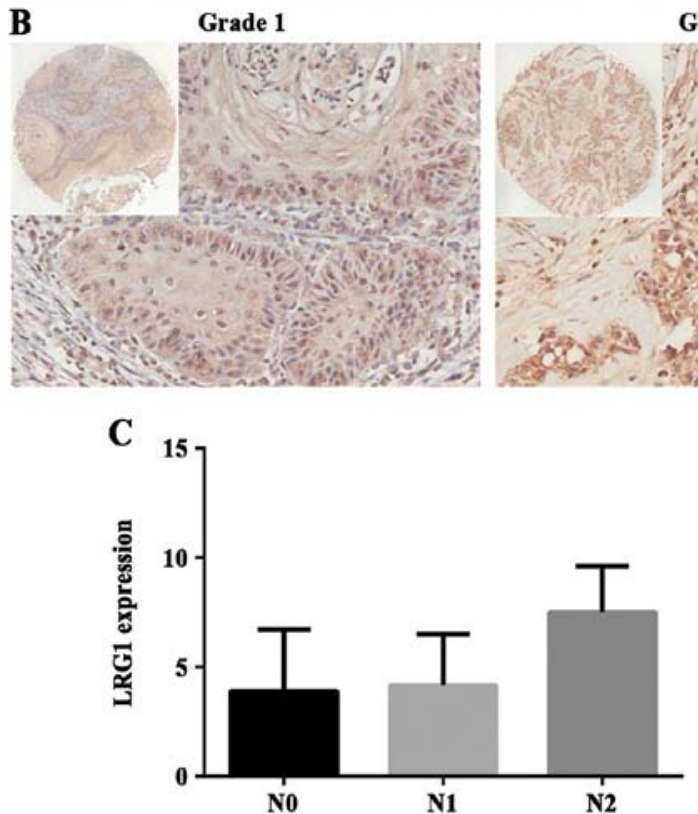

N1

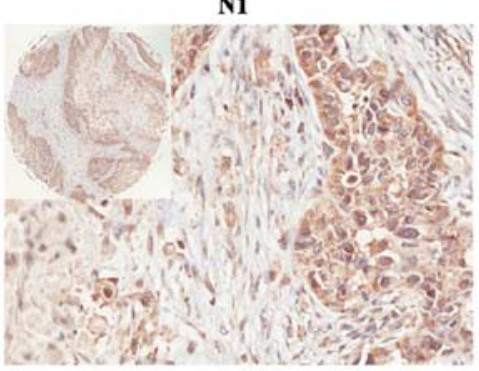

Grade 2

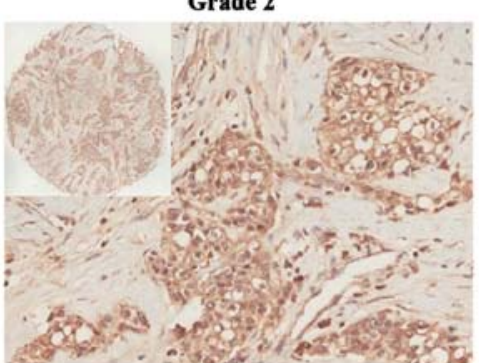

N2

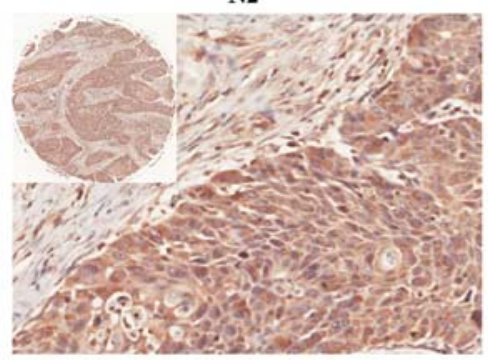

Grade 3

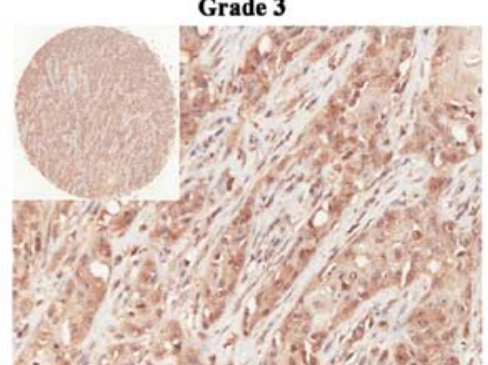

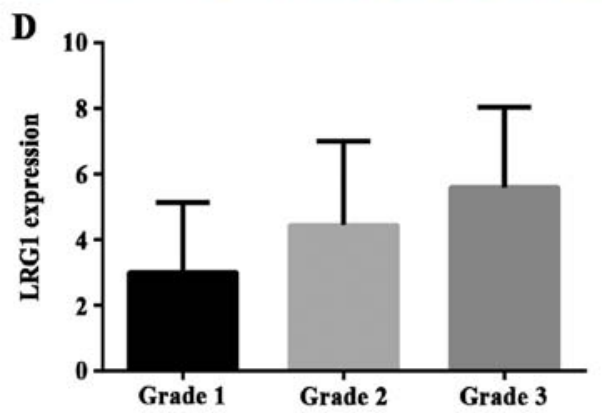

Figure 4. LRG1 protein expression in different $\mathrm{N}$ stages and malignancy grades in the HNSCC TMA. (A and B) Representative images of IHC staining, respectively, in three $\mathrm{N}$ stage groups and in three malignant grade groups (magnification, $\mathrm{x} 400$ ). In the top left corner are original microscopic images at a magnification of $\mathrm{x} 100$. (C and D) Graphical representation of the mean \pm SD data representing CES of LRG1 protein staining presented in A and B, respectively. There was no statistically significance between $\mathrm{N}$ stages or tumor grades; $\mathrm{P}>0.05$.

A
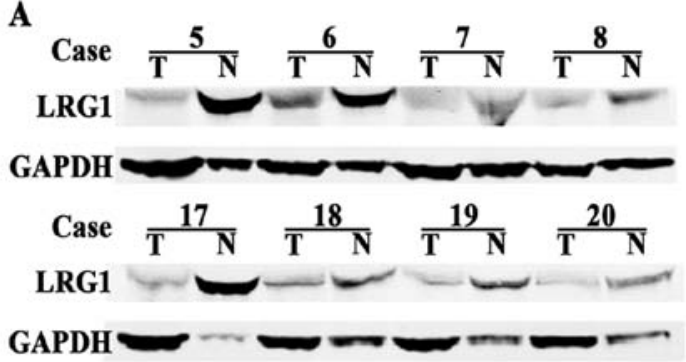

C

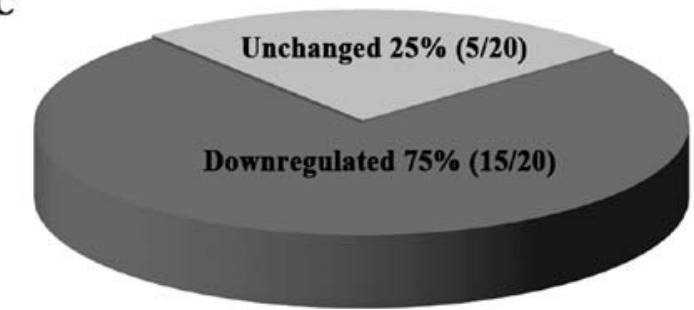

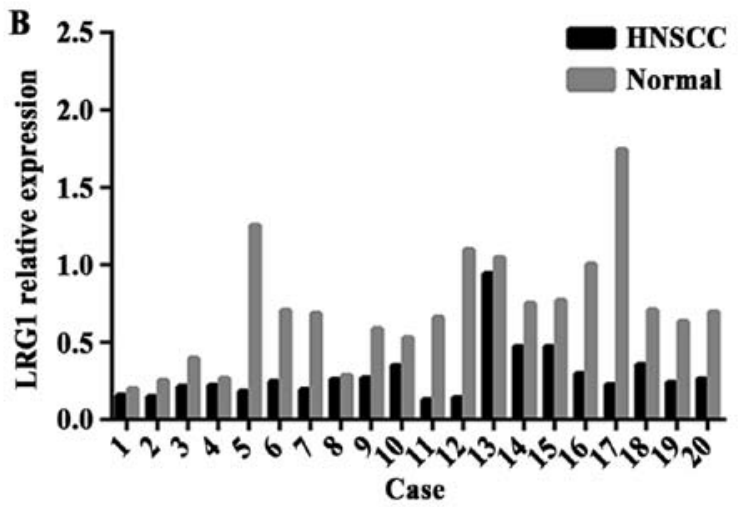

Case

Figure 5. LRG1 expression is reduced in HNSCC tissues. (A) Western blot analysis of LRG1 protein level in eight paired HNSCC (T) and adjacent noncancerous tissues (N) (other pairs were not shown). (B) A graphical representation of the mean data represents relative density of LRG1 to GAPDH in all 20 paired samples. t-test was used to analyze LRG1 expression of each pair. There was no significant difference in cases 1, 2, 4, 8 and 13. (C) Proportions of downregulation and no change are indicated in dark and light grey colors in the pie chart, respectively. 
gender $(\mathrm{P}=0.2027)$, age $(\mathrm{P}=0.3387)$ and location $(\mathrm{P}=0.6628)$. In addition, in regards to the clinical stages, LRG1 expression in the early stages was negatively different from that in the advanced stages $(\mathrm{P}=0.1710)$. The results of the analysis are summarized in Table II.

LRG1 expression is reduced in the HNSCC tissues. The above results were confirmed by IHC staining. In addition, we examined the level of LRG1 protein in 20 pairs of HNSCC and adjacent non-cancerous tissues. The LRG1 protein level was downregulated in the HNSCC tissues compared with that in the para-carcinoma tissues, and a representative portion of the western blot findings are shown in Fig. 5A. Transformed pillar and pie charts are presented in Fig. 5B and C, showing a significant decrease in LRG1 expression in 75\% (15/20) of the HNSCC patients.

\section{Discussion}

It has been reported that the expression of LRG1 is dysregulated in various types of malignant tumors, yet its role in HNSCC has not been addressed. The present study found that the LRG1 gene was downregulated in HNSCC tissues first based on analysis of the GEO database. Similar results were demonstrated at the protein level from evaluation of the tissues collected and the TMA. In addition, the expression level of LRG1 was negatively associated with several clinicopathological features. These findings uncovered that LRG1 may play a role in HNSCC tumorigenesis and potentially offers clinical value in early diagnosis.

The term head and neck carcinoma covers all malignant tumors arising in the nasal and oral cavities, pharynx, larynx and the paranasal sinuses. Most are squamous cell carcinomas. Compared with other cancers such as breast, cervix and colorectal, patients with HNSCC have relatively poor prognosis after diagnosis $(3,21)$. The reason is related to a failure in identifying effective measurements for diagnosis at the early stage. According to tumor-node-metastasis (TNM) classification and clinical staging, treatment procedures differ substantially between early- and late-stage HNSCC. Earlystage HNSCC patients receive minimally invasive surgery or irradiation alone due to the small tumor size with least lymphatic and hematogenous spread, and benefit from removal of the neoplasia. However, lack of appropriate screening biomarkers leads to difficulty in early diagnosis. Biomarkers with promising potential reflecting physical changes are in urgent need for clinical application.

LRG1 is considered as a candidate biomarker of tumor detection as it was found aberrantly (particularly elevated) expressed in different types of cancers or other body fluids vs. normal unmutated cells. Wu et al (22) found that expression of LRG1 was elevated in serum and other histological subtypes of early and late stage epithelial ovarian cancer cases. Thus, LRG1 is a potential biomarker alone or in combination with CA125 for the diagnosis of ovarian cancer. LRG1 was also found to be expressed at higher levels in urinary exosomes and lung tissue of non-small cell lung cancer patients (23). Overexpression of LRG1 was interpreted as its auxo-action on angiogenesis, cell migration and invasion $(12,24,25)$. It was found to bind to the accessory receptor endoglin, causing a switch in TGF- $\beta$ signaling in endothelial cells via the ALK-1/Smad1/5/8 pathway, which led to the promotion of neovascularization (12). Moreover, LRG activated TGF- $\beta$ signaling by upregulating TGF- $\beta 1$ and promoting the phosphorylation of its downstream smad proteins, which was associated with enhanced migration and invasion of glioma cells (26).

However, contrary to the above mentioned studies, in our research LRG1 expression was decreased at both the gene and protein level tested in the GEO database analysis, IHC and immunoblotting in HNSCC tissues, implying LRG1 as a tumor suppressor. Similar results were found in hepatocellular carcinoma, endometrial carcinoma and Lewis lung carcinoma cell lines $(15,27,28)$. We may attribute the discrepancy of LRG1 function in certain types of cancers to its tissue specificity, while the underlying mechanism needs to be understood. A recent study showed that LRG1 enhanced TGF- $\beta 1$-smad2-induced growth inhibition and apoptosis in Lewis lung carcinoma and Hep3B cells which lacked endoglin (28). However, the function of LRG1 in the tumorigenesis of HNSCC needs further investigation.

The degree of tumor malignancy is determined by several factors such as proliferation, cell cycle and microvessels in neoplasms (29-31). It has been reported that LRG1 is expressed higher in grade IV glioma cell lines than that in grade III glioma cells and LRG1 silencing was found to lead to cell cycle arrest with the accumulation of cells in the $\mathrm{G} 0 / \mathrm{G} 1$ phase and reduced cell numbers in the $S$ and G2/M phases (32). Moreover, downregulation of cell cycle genes including cyclin B, D1 and E by LRG1 silencing was observed, implying that LRG1 may regulate the cell cycle of glioblastoma cells through these cyclins. However, in the present study, we found that LRG1 expression had no significant difference among the three grades of HNSCC by GEO database analysis. Then we observed the LRG1 protein staining in different tumor grades, and the same trend was noted. Low expression of LRG1 was observed in HNSCC due to the fact that its tissue characteristics are different from glioblastoma as referred above. However, it is perplexing that lower LRG1 expression was not present in a higher grade of HNSCC. This is a focus in our following experiments.

It has been referred in various studies that LRG is involved in the suppression of tumor invasion and migration. In hepatocellular carcinoma cells, overexpression and knockdown of LRG1, respectively, weakened and strengthened the capability of tumor cell migration and invasion (27), which can also explain the downregulated expression of LRG1 in HNSCC in the present study. However, the LRG1 expression level was negatively associated with HNSCC stage and lymphatic metastasis. In our observation, GEO database analysis and IHC staining showed that there was no significant difference in LRG1 expression between $\mathrm{T}$ or $\mathrm{N}$ stages. This implies that LRG1 in HNSCC is involved in tumorigenesis only, not in progression.

In conclusion, our findings first demonstrated that LRG1 expression is downregulated in HNSCC, and this suppressed expression was negatively associated with tumor differentiation, tumor stage and local lymph node metastasis, implying that LRG1 is involved in tumorigenesis, but not in the development of HNSCC. These findings may provide potential clinical value for the early diagnosis of HNSCC. 


\section{Acknowledgements}

The present study was supported by grants from the National Natural Science Foundation of China (no. 81372880), and the Natural Science Foundation of Hubei Province (no. 2012FFA045).

\section{References}

1. Ota I, Okamoto N, Yane K, Takahashi A, Masui T, Hosoi H and Ohnishi T: Therapeutic strategies for head and neck cancer based on p53 status. Exp Ther Med 3: 585-591, 2012.

2. Ozdek A, Sarac S, Akyol MU, Unal OF and Sungur A: Histopathological predictors of occult lymph node metastases in supraglottic squamous cell carcinomas. Eur Arch Otorhinolaryngol 257: 389-392, 2000.

3. Jemal A, Siegel R, Ward E, Hao Y, Xu J, Murray T and Thun MJ: Cancer statistics, 2008. CA Cancer J Clin 58: 71-96, 2008.

4. Wang L, Chen C, Li F, Hua Q, Chen S, Xiao B, Dai M, Li M, Zheng A, Yu D, et al: Down-regulation of neutrophil gelatinase-associated lipocalin in head and neck squamous cell carcinoma correlated with tumorigenesis, not with metastasis. Int J Clin Exp Pathol 8: 8857-8868, 2015

5. Koole K, van Kempen PM, Swartz JE, Peeters T, van Diest PJ, Koole R, van Es RJ and Willems SM: Fibroblast growth factor receptor 3 protein is overexpressed in oral and oropharyngeal squamous cell carcinoma. Cancer Med 5: 275-284, 2016.

6. Haupt $\mathrm{H}$ and Baudner S: Isolation and characterization of an unknown, leucine-rich 3.1-S-alpha2-glycoprotein from human serum (author's transl). Hoppe Seylers Z Physiol Chem 358: 639-646, 1977 (In German).

7. Kobe B and Kajava AV: The leucine-rich repeat as a protein recognition motif. Curr Opin Struct Biol 11: 725-732, 2001.

8. Buchanan SG and Gay NJ: Structural and functional diversity in the leucine-rich repeat family of proteins. Prog Biophys Mol Biol 65: 1-44, 1996.

9. Serada S, Fujimoto M, Terabe F, Iijima H, Shinzaki S, Matsuzaki S, Ohkawara T, Nezu R, Nakajima S, Kobayashi T, et al: Serum leucine-rich alpha-2 glycoprotein is a disease activity biomarker in ulcerative colitis. Inflamm Bowel Dis 18: 2169-2179, 2012

10. Kharbanda AB, Rai AJ, Cosme Y, Liu K and Dayan PS: Novel serum and urine markers for pediatric appendicitis. Acad Emerg Med 19: 56-62, 2012.

11. Serada S, Fujimoto M, Ogata A, Terabe F, Hirano T, Iijima H, Shinzaki S, Nishikawa T, Ohkawara T, Iwahori K, et al: iTR AQ-based proteomic identification of leucine-rich alpha-2 glycoprotein as a novel inflammatory biomarker in autoimmune diseases. Ann Rheum Dis 69: 770-774, 2010.

12. Wang X, Abraham S, McKenzie JA, Jeffs N, Swire M, Tripathi VB, Luhmann UF, Lange CA, Zhai Z, Arthur HM, et al: LRG1 promotes angiogenesis by modulating endothelial TGF- $\beta$ signalling. Nature 499: 306-311, 2013.

13. He X, Wang Y, Zhang W, Li H, Luo R, Zhou Y, Li C, Liao M, Huang H, Lv X, et al: Screening differential expression of serum proteins in AFP-negative HBV-related hepatocellular carcinoma using iTRAQ -MALDI-MS/MS. Neoplasma: Sep 20, 2013 (Epub ahead of print). doi: 10.4149/neo_2014_001.

14. Wu J, Xie X, Nie S, Buckanovich RJ and Lubman DM: Altered expression of sialylated glycoproteins in ovarian cancer sera using lectin-based ELISA assay and quantitative glycoproteomics analysis. J Proteome Res 12: 3342-3352, 2013.

15. Wen SY, Zhang LN, Yang XM, Zhang YL, Ma L, Ge QL, Jiang SH, Zhu XL, Xu W, Ding WJ, et al: LRG1 is an independent prognostic factor for endometrial carcinoma. Tumour Biol 35: $7125-7133,2014$

16. Uen YH, Lin KY, Sun DP, Liao CC, Hsieh MS, Huang YK, Chen YW, Huang PH, Chen WJ, Tai CC, et al: Comparative proteomics, network analysis and post-translational modification identification reveal differential profiles of plasma Con A-bound glycoprotein biomarkers in gastric cancer. J Proteomics 83: 197-213, 2013.
17. Wu RS, Yu CS, Liu KC, Huang HY, Ip SW, Lin JP, Chueh FS, Yang JS and Chung JG: Citosol (thiamylal sodium) triggers apoptosis and affects gene expressions of murine leukemia RAW 264.7 cells. Hum Exp Toxicol 31: 771-779, 2012.

18. Ladd JJ, Busald T, Johnson MM, Zhang Q, Pitteri SJ, Wang H, Brenner DE, Lampe PD, Kucherlapati R, Feng Z, et al: Increased plasma levels of the APC-interacting protein MAPRE1, LRG1, and IGFBP2 preceding a diagnosis of colorectal cancer in women. Cancer Prev Res 5: 655-664, 2012.

19. Kakisaka T, Kondo T, Okano T, Fujii K, Honda K, Endo M, Tsuchida A, Aoki T, Itoi T, Moriyasu F, et al: Plasma proteomics of pancreatic cancer patients by multi-dimensional liquid chromatography and two-dimensional difference gel electrophoresis (2D-DIGE): Up-regulation of leucine-rich alpha-2-glycoprotein in pancreatic cancer. J Chromatogr B Analyt Technol Biomed Life Sci 852: 257-267, 2007.

20. Lindén M, Segersten U, Runeson M, Wester K, Busch C, Pettersson U, Lind SB and Malmström PU: Tumour expression of bladder cancer-associated urinary proteins. BJU Int 112: 407-415, 2013

21. Jemal A, Thun MJ, Ries LA, Howe HL, Weir HK, Center MM, Ward E, Wu XC, Eheman C, Anderson R, et al: Annual report to the nation on the status of cancer, 1975-2005, featuring trends in lung cancer, tobacco use, and tobacco control. J Natl Cancer Inst 100: 1672-1694, 2008.

22. Wu J, Yin H, Zhu J, Buckanovich RJ, Thorpe JD, Dai J, Urban N and Lubman DM: Validation of LRG1 as a potential biomarker for detection of epithelial ovarian cancer by a blinded study. PLoS One 10: e0121112, 2015.

23. Li Y, Zhang Y, Qiu F and Qiu Z: Proteomic identification of exosomal LRG1: A potential urinary biomarker for detecting NSCLC. Electrophoresis 32: 1976-1983, 2011.

24. Lynch J, Meehan MH, Crean J, Copeland J, Stallings RL and Bray IM: Metastasis suppressor microRNA-335 targets the formin family of actin nucleators. PLoS One 8: e78428, 2013.

25. Lynch J, Fay J, Meehan M, Bryan K, Watters KM, Murphy DM and Stallings RL: MiRNA-335 suppresses neuroblastoma cell invasiveness by direct targeting of multiple genes from the non-canonical TGF- $\beta$ signalling pathway. Carcinogenesis 33: 976-985, 2012

26. Zhong D, He G, Zhao S, Li J, Lang Y, Ye W, Li Y, Jiang C and Li X: LRG1 modulates invasion and migration of glioma cell lines through TGF- $\beta$ signaling pathway. Acta Histochem 117: 551-558, 2015.

27. Zhang Y, Luo Q, Wang N, Hu F, Jin H, Ge T, Wang C and Qin W: LRG1 suppresses the migration and invasion of hepatocellular carcinoma cells. Med Oncol 32: 146, 2015.

28. Takemoto N, Serada S, Fujimoto M, Honda H, Ohkawara T, Takahashi T, Nomura S, Inohara $\mathrm{H}$ and Naka T: Leucine-rich $\alpha$-2-glycoprotein promotes TGF $\beta 1$-mediated growth suppression in the Lewis lung carcinoma cell lines. Oncotarget 6: 11009-11022, 2015.

29. Schonberg DL, Lubelski D, Miller TE and Rich JN: Brain tumor stem cells: Molecular characteristics and their impact on therapy. Mol Aspects Med 39: 82-101, 2014.

30. Cavazza A, Corradini C, Marini M, Roda LG and Valenti A: Capillary electrophoresis coupled with mass spectrometry for the evaluation of substance $\mathrm{P}$ enzymatic degradation by SaOS-2 human osteosarcoma. J Chromatogr B Analyt Technol Biomed Life Sci 879: 2501-2506, 2011.

31. Sulzbacher I, Birner P, Dominkus M, Pichlhofer B and Mazal PR: Expression of platelet-derived growth factor-alpha receptor in human osteosarcoma is not a predictor of outcome. Pathology 42: 664-668, 2010

32. Zhong D, Zhao S, He G, Li J, Lang Y, Ye W, Li Y, Jiang C and Li X: Stable knockdown of LRG1 by RNA interference inhibits growth and promotes apoptosis of glioblastoma cells in vitro and in vivo. Tumour Biol 36: 4271-4278, 2015. 